Florida International University FIU Digital Commons

\title{
Detection of Modality-Specific Properties in Unimodal and Bimodal Events during Prenatal Development
}

Jimena Vaillant

Florida International University, jvail001@fiu.edu

DOI: $10.25148 /$ etd.FI10112012

Follow this and additional works at: https://digitalcommons.fiu.edu/etd

\section{Recommended Citation}

Vaillant, Jimena, "Detection of Modality-Specific Properties in Unimodal and Bimodal Events during Prenatal Development" (2010). FIU Electronic Theses and Dissertations. 303.

https://digitalcommons.fiu.edu/etd/303 


\section{FLORIDA INTERNATIONAL UNIVERSITY}

Miami, Florida

DETECTION OF MODALITY-SPECIFIC PROPERTIES IN UNIMODAL AND

BIMODAL EVENTS DURING PRENATAL DEVELOPMENT

A thesis submitted in partial fulfillment of the

requirements for the degree of

MASTER OF SCIENCE

in

PSYCHOLOGY

by

Jimena Vaillant

2010 
To: Dean Kenneth Furton

College of Arts and Sciences

This thesis, written by Jimena Vaillant, and entitled Detection of Modality-Specific Properties in Unimodal and Bimodal Events during Prenatal Development, having been approved in respect to style and intellectual content, is referred to you for judgment.

We have read this thesis and recommend that it be approved.

Mary Levitt

$\overline{\text { Lorraine E. Bahrick }}$

Robert Lickliter, Major Professor

Date of Defense: September 1, 2010

The thesis of Jimena Vaillant is approved.

\begin{tabular}{r}
$\begin{array}{r}\text { Dean Kenneth Furton } \\
\text { College of Arts and Sciences }\end{array}$ \\
\hline Interim Dean Kevin O’Shea \\
University Graduate School
\end{tabular}

Florida International University, 2010 


\section{ACKNOWLEDGMENTS}

I am incredibly thankful to my supervisor and mentor, Dr. Robert Lickliter, for his guidance, support, and encouragement throughout this process. I am also grateful to the MBRS RISE program for providing the resources that have helped me to complete my project and enhance my research experience. Lastly, I offer my regards to my family and friends for their understanding and support during this time. 


\title{
ABSTRACT OF THE THESIS \\ DETECTION OF MODALITY-SPECIFIC PROPERTIES IN UNIMODAL AND BIMODAL EVENTS DURING PRENATAL DEVELOPMENT
}

\author{
by
}

\section{Jimena Vaillant}

Florida International University, 2010

Professor Robert Lickliter, Major Professor

Predictions of the Intersensory Redundancy Hypothesis (IRH) state that early in development information presented to a single sense modality (unimodal) selectively recruits attention to and enhances perceptual learning of modality-specific properties of stimulation at the expense of amodal properties, while information presented redundantly across two or more modalities (bimodal) results in enhanced perceptual learning of amodal properties. The present study explored these predictions during prenatal development by assessing bobwhite quail embryos’ detection of pitch, a modalityspecific property, under conditions of unimodal and redundant bimodal stimulation. Chicks’ postnatal auditory preferences between the familiarized call and the same call with altered pitch were assessed following hatching. Unimodally-exposed chicks significantly preferred the familiarized call over the pitch-modified call, whereas bimodally-exposed chicks did not prefer the familiar call over the pitch-modified call. Results confirm IRH predictions, demonstrating unimodal exposure facilitates learning of modality-specific properties, whereas redundant bimodal stimulation interferes with learning of modality-specific properties. 


\section{TABLE OF CONTENTS}

CHAPTER $\quad$ PAGE

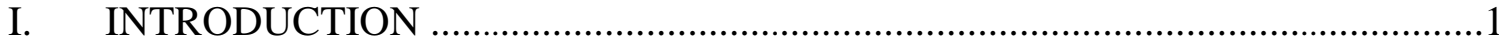

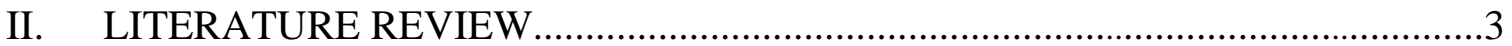

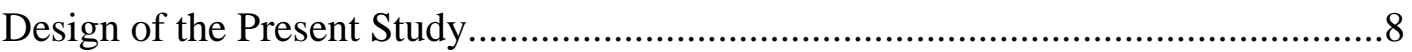

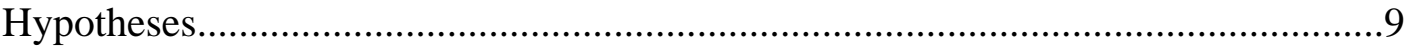

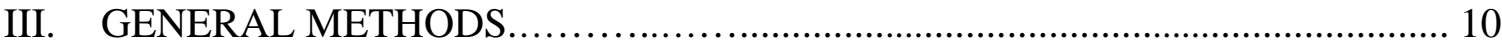

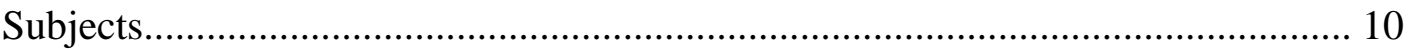

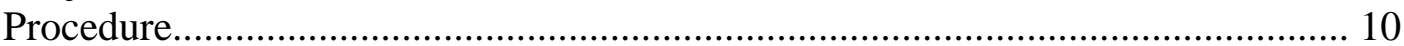

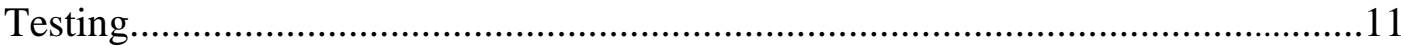

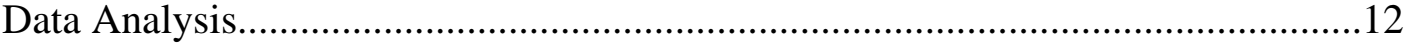

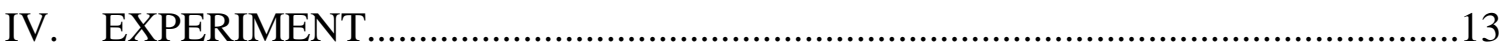

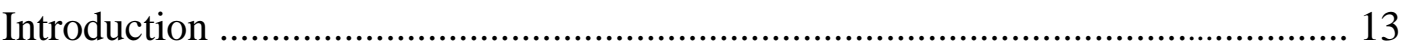

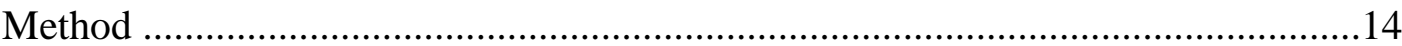

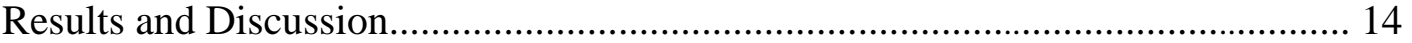

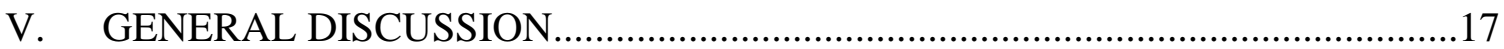

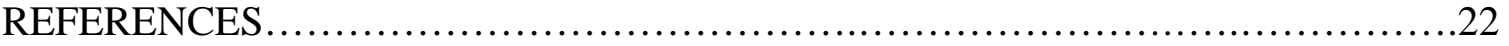




\section{CHAPTER I.}

\section{Introduction}

Research has consistently demonstrated that young infants readily perceive multimodal information in the weeks and months following birth (see Lewkowicz \& Lickliter, 1994; Rose \& Ruff, 1987 for reviews). For example, 3-6 month-old infants are capable of matching soundtracks to the appropriate object hitting a surface based on the object’s material and composition (Bahrick, 1983, 1987, 1988, 1992). Moreover, 5-7 month-old infants are able to match voices and faces on the basis of the speaker's age, gender, and affective expression (Bahrick, Netto, \& Hernandez-Reif, 1998; Walker, 1982; Walker-Andrews, Bahrick, Raglioni, \& Diaz, 1991). Understanding how infants come to perceive and obtain meaning from the flux of multimodal stimulation available in their physical and social environments is critical to ultimately understanding how perception develops.

According to E. J. Gibson’s (1969) invariant detection view, infants are able to differentiate objects and events by detecting invariants, structural properties in the environment which are stable and do not change with changes in illumination or transformations from movement of the observer or the object itself. The detection of invariant relations is thought to allow relatively naïve perceivers to quickly distinguish meaningful properties of objects and events. Consistent with Gibson’s view, Bahrick \& Pickens (1994) argued that the detection of amodal properties of stimulation initially guides perceptual learning. Amodal information is information that is redundant across two or more sense modalities. The dimensions of time, space, and intensity can be conveyed by multiple senses. For instance, the sound and sight of a bouncing ball are 
synchronous, derive from the same location, and share the same rate, rhythm, and intensity. Nearly all events provide amodal information because they take place across time, are dispersed in space, and have particular intensity patterns.

How young organisms extract and perceive information from the environment during early development is vital to understanding how perception of multimodal events develops. Studies that manipulate sensory experience during prenatal and early postnatal development are difficult to carry out with human subjects, and the use of animal models to explore the experiential factors contributing to early perceptual development has proven important to advancing knowledge in this area (Lickliter \& Bahrick, 2000). For example, various studies using precocial birds suggest that modifying sensory experience during the perinatal period can have significant effects on early perceptual development and perceptual learning (Gottlieb, 1971, 1993; Lickliter and Stoumbos, 1991; Honeycutt \& Lickliter, 2002; reviewed in Lickliter, 2005). For example, a study by Lickliter and Lewkowicz (1995) indicated that a certain range of prenatal sensory stimulation is necessary to foster species-typical perceptual capabilities. Bobwhite quail chicks deprived of vestibular and tactile stimulation during the late prenatal period failed to show typical perceptual functioning. Chicks incubated in isolation during the late stages of prenatal development responded to auditory cues into later stages of postnatal development, failed to respond to visual cues at ages chicks incubated in groups display visual receptiveness, and failed to show auditory learning of a maternal call, which is typically seen in embryos incubated in groups. Despite such findings, the role of unimodal and multimodal sensory experience in maintaining, facilitating, or interfering with the typical course of intersensory development remains to be fully explored. 


\section{CHAPTER II.}

\section{Literature Review}

Numerous studies have shown that both animal and human infants are adept perceivers of amodal information (see Lewkowicz \& Lickliter, 1994; Gibson \& Pick, 2000 for reviews). One of the earliest and most basic amodal properties detected by infants is temporal synchrony (Lewkowicz, 2000). Research has shown that amodal properties are detected developmentally prior to modality-specific properties of events (Bahrick 1992, 1994, 2001; Lewkowicz, 2000). For example, infants can perceive temporal properties of stimulation such as synchrony, rhythm, and tempo (Spelke, 1979; Bahrick, 1983, 1987, 1988; Lewkowicz, 2000). Similarly, they can detect the spatial association of objects and their corresponding sounds (Bahrick, 1992; Morrongiello, 1994). Redundantly specified amodal information is particularly salient to human and nonhuman infants (Lewkowicz, 2000; Bahrick \& Lickliter, 2000, 2002). For instance, 7month-old infants, but not 3- or 5-month-old infants, detect the arbitrary relation between the color-shape of an object and the pitch of its impact sounds when the sounds and sights of impacts are synchronous (Bahrick, 1994). In several studies assessing infants ranging from 4 to 10 months old, Lewkowicz (1988a, 1988b, 1992, 1994, 1996) demonstrated that infants distinguish changes in amodal properties such as rate and duration when the changes occurred concurrently and synchronously in both the auditory and visual sensory modalities. When these changes occurred in only one modality, infants were not able to consistently demonstrate this discrimination. Specifically, 10-month old infants habituated to an inanimate auditory/visual compound stimulus did not respond to a change that occurred solely in the visual component during testing trials; similarly, 4- 
month old infants habituated to a visible and auditory face of a person speaking did not respond to changes that occurred only in the audible component (i.e., human voice) (Lewkowicz, 1988b, 1996).

Despite the results of various studies assessing the perception of amodal relations during early development, most theories of perceptual development do not explain how intersensory development actually proceeds. Thus, current research still leaves unanswered exactly how infants are able to guide their attention to certain aspects of stimulation and ignore others. It is this selective attention (i.e., tendency to orient toward, or process particular information to the exclusion of other information) that allows young organisms to efficiently attend to relevant information in their immediate environment. The senses are constantly picking up information from the external world that is perceived through seeing, hearing, touching, tasting, and smelling. The individual then has to selectively attend to certain aspects of stimulation while ignoring others. This ability is crucial for typical development. For example, listening carefully to one person speaking while ignoring other conversations in the same room is necessary for effective social communication. Attending to specific features of stimulation provides extensive experience with that class of features, allowing for further processing and learning. For example, selective attention to faces and the arrangement of their features is necessary for the infant to learn to distinguish between different faces, to recognize and differentiate expressions and affect, to learn language through mouth movements, and to engage in appropriate social exchanges. Nearly all events in the environment provide both redundant amodal information (e.g. intensity of facial expression) and nonredundant modality-specific information (e.g. color of an individual’s hair). However, relatively 
little is known about how perception of amodal information (i.e., tempo, rhythm) is coordinated with perception of modality-specific information (i.e., color, timbre) and how it drives selective attention during early development in an environment that offers continuous fluctuations of multimodal and unimodal stimulation from events and objects.

Bahrick \& Lickliter $(2000$, 2002) have proposed a model of selective attention, the Intersensory Redundancy Hypothesis (IRH), to explain how and under what conditions selective attention and perceptual processing are promoted to different aspects or properties of events (amodal versus modality-specific) during early development. Intersensory redundancy refers to the temporally synchronous and spatially collocated occurrence of the same information (e.g., rate, rhythm, duration, intensity shifts) across two or more senses. According to the IRH, intersensory redundancy is highly salient and directs selective attention to amodal aspects of events that are redundantly specified across the senses, at the expense of nonredundantly specified information within the same event, particularly during early development. This, in turn, promotes perception, learning, and memory for amodal information (Bahrick \& Lickliter, 2000, 2002).

The IRH makes four specific and testable predictions: 1) Redundant multimodal stimulation selectively recruits attention and promotes perceptual processing of the amodal properties of events (at the expense of non-redundantly specified properties) to a greater extent than does unimodal (e.g., visual or auditory) stimulation. This phenomenon is termed intersensory facilitation. 2) Information presented nonredundantly or to one sense modality alone selectively recruits attention and promotes perceptual processing of modality-specific properties of stimulation to a greater extent than does multimodal stimulation. This phenomenon is termed unimodal facilitation. 
This occurs in part because redundancy is not available to compete for attention in unimodal stimulation. 3) As attention becomes more efficient and flexible with experience, detection of both amodal and modality-specific properties emerges in both redundant, multimodal and nonredundant, unimodal stimulation. 4) When cognitive load is high and attentional resources are strained, as in tasks of high difficulty in relation to the perceiver's expertise, intersensory and unimodal facilitation occur across development (Bahrick \& Lickliter, in press). The third and fourth predictions are developmental predictions of the IRH because they refer to implications for perceptual learning across the lifespan.

A variety of studies from both human and non-human animal infants have supported the first two predictions of the IRH. Intersensory facilitation of temporal properties of events has been demonstrated in human infants and bobwhite quail chicks. For example, rhythm and tempo are perceived more readily by 3-month-old infants when they are conveyed through two sense modalities rather than through one modality (Bahrick \& Lickliter, 2000; Bahrick, Flom \& Lickliter, 2002). Animal studies have also provided further support for the first prediction of the IRH. Quail embryos are able to learn a maternal call significantly faster when the rate, rhythm, and duration of the call is synchronized with a light, thus providing intersensory redundancy, than when the call is presented alone (Lickliter, Bahrick, \& Honeycutt, 2002). Further, Lickliter, Bahrick, and Honeycutt (2004) found that chicks receiving redundant bimodal stimulation of the temporal features of the maternal call prenatally remembered the call four times longer into postnatal development than chicks receiving prenatal unimodal exposure. 
Research also indicates that such intersensory facilitation results from the redundancy and not to other factors such as a greater amount of stimulation in two modalities as compared with one. For example, intersensory facilitation is not found under conditions of multimodal stimulation where there is no redundancy (e.g., asynchronous but congruent auditory and visual patterns, see Bahrick \& Lickliter, 2000; Bahrick, Flom, \& Lickliter, 2002; Flom \& Bahrick, 2007; Lickliter, Bahrick, \& Honeycutt, 2002).

Evidence of unimodal facilitation has also been found in studies of human infants. For example, infants show heightened discrimination and memory for the orientation of a toy hammer tapping in unimodal visual and in asynchronous audiovisual stimulation, where no redundancy is available. In contrast, when redundancy is available in synchronous, audiovisual stimulation, infants fail to discriminate a change in orientation (Bahrick, Lickliter, \& Flom, 2006). A study with 2 month-old infants showed that face discrimination (based on modality-specific properties) is facilitated when the face is presented unimodally, but not under bimodal synchronous conditions (Bahrick, Lickliter, Vaillant, Schuman, \& Castellanos, 2004). Research has also revealed that face-voice redundancy impairs face discrimination rather than simply the presence of stimulation in a second sense modality or a greater quantity of stimulation (Vaillant-Molina, Newell, Castellanos, Bahrick, \& Lickliter, 2006). These findings support the second prediction of the IRH and indicate that modality-specific properties are enhanced in unimodal stimulation and attenuated in multimodal stimulation, where salient redundant amodal properties compete for infants' attention. Because selective attention is basic to what is 
perceived and learned, the dynamics of early selectivity likely has lasting effects on the development of perceptual and learning abilities across infancy and beyond.

Perception has been described as a process whereby responsiveness to perceptual information based on its saliency develops into increasingly finer differentiation of stimulus features (Gibson, 1969). The IRH proposes that the saliency of intersensory redundancy promotes attention to some properties of stimulation at the expense of others, which in turn, provides the basis for what is perceived and learned. Hence, the term attention here refers to the perceptual process through which organisms select specific kinds of information from all the information available in their environment.

\section{Design of the Present Study}

The current study uses animal subjects to determine if prenatal unimodal exposure facilitates learning of modality-specific properties of stimulation, and whether prenatal redundant bimodal stimulation interferes with attention to and learning of modality-specific properties of stimulation. As previously reviewed, postnatal studies with human infants have supported this prediction with the use of faces, voices, and orientation of objects. This study uses an animal model, the bobwhite quail, to investigate the second prediction of the Intersensory Redundancy Hypothesis. As discussed by Lickliter (2000), comparative studies can provide insight into developmental mechanisms and processes that are involved in the development of perception. Experimental manipulations of sensory experience are severely limited with human subjects, and comparative animal research permits experimental modifications of sensory experience, including prenatal sensory augmentation, deprivation, and displacement, thereby 
allowing for the analysis of organismic and environmental factors contributing to early perceptual development.

The present study used bobwhite quail embryos and chicks to compare unimodal and multimodal perception during the late stages of prenatal development. Bobwhite quail embryos were exposed to an individual bobwhite maternal call either unimodally (auditory only) or bimodally (redundant audio-visual stimulation) on the day prior to hatch. Following hatching, chicks were tested individually between the familiar version of the maternal call that was presented prenatally (i.e., no acoustic changes) versus the same maternal call with an altered pitch range (all other acoustic features held constant). Hypotheses

It was hypothesized that prenatal unimodal auditory exposure would facilitate learning of the modality-specific property of pitch, whereas redundant audio-visual exposure would interfere with attention to and learning of the modality-specific property of pitch. According to the predictions of the Intersensory Redundancy Hypothesis, this should be the case because only embryos receiving unimodal exposure to the maternal call focus their selective attention on modality-specific stimulus properties during familiarization, whereas embryos receiving redundant bimodal exposure focus their attention on amodal stimulus properties such as rhythm, rate, or duration of the call, and thus should not detect the pitch change during postnatal testing. 


\section{CHAPTER III.}

\section{General Methods}

\section{Subjects}

Subjects were 141 incubator reared bobwhite quail chicks (Colinus virginianus). Fertilized unincubated eggs were received weekly from a commercial supplier and set in a BSS-160 Grumbach Incubator maintained at 75-80\% relative humidity and $37.5^{\circ} \mathrm{C}$. Embryonic age was calculated on the basis of the first day of incubation as Day 0, and so forth. To control for potential variations in developmental age, only those birds that hatched on Day 23 were used as subjects. To control for possible differences between batches, subjects for each condition were selected from at least three different batches of eggs. Following hatching, groups of 15-20 subjects were housed in a rearing tub until testing. Chicks were given constant access to food and water, except during testing sessions. Ambient air temperature was maintained at approximately $30^{\circ} \mathrm{C}$.

Procedure

Approximately 24 hrs prior to hatching, embryos were transferred to a sound attenuated stimulation room and placed in a portable hatcher, maintained at approximately $37.5^{\circ} \mathrm{C}$ and $80 \%$ relative humidity. This hatcher allowed embryos to receive audio-visual stimulation via a transparent plastic window located directly above the embryos. Audio-visual stimulus presentations were delivered by means of a computer with a custom designed software program running a speaker that broadcast the maternal call. The speaker was placed on top of a small opening at the top of the hatcher. The computer software program also ran an amplifier connected to an adjustable desk lamp that transmitted the pulsed light synchronously with the notes of the call. In this 
condition, the synchronized light provided the same amodal information (rhythm, rate, and duration) as the five notes of the maternal call. The lamp was placed directly above the portable hatcher window.

Bobwhite quail embryos were divided into 2 experimental conditions: (1) a Unimodal Auditory group $(n=49)$ exposed to an individual variant of the bobwhite maternal call for $10 \mathrm{~min} / \mathrm{hr}$ during the $24 \mathrm{hr}$ prior to hatching, (2) a Bimodal AudioVisual group $(\mathrm{n}=51)$ exposed to an individual variant of the bobwhite maternal call paired with a pulsing light temporally synchronized with the notes of the call for 10 $\mathrm{min} / \mathrm{hr}$ for the $24 \mathrm{hr}$ prior to hatching. A third group served as controls $(\mathrm{n}=41)$ and received no supplemental prenatal sensory stimulation. Subjects in all groups were transferred to rearing tubs immediately after hatching and were housed there in groups of same-aged chicks until testing at $24 \mathrm{hr}$ following hatching.

Testing

Postnatal behavioral tests were conducted in an arena $130 \mathrm{~cm}$ in diameter, encircled by a wall $60 \mathrm{~cm}$ in height. The arena surface was painted black, and an opaque black curtain covered the wall of the arena. A video camera mounted directly above the arena allowed for remote observation and data collection. Two semi-circular approach areas each comprising approximately $5 \%$ of the total area of the testing arena were demarcated on a remote video monitor. Both approach areas contained a small speaker mounted to the arena wall and hidden behind the black curtain to allow for the presentation of auditory stimuli during the testing trials.

All chicks were tested individually between the familiar version of the maternal call that was presented prenatally versus the same maternal call altered to a one-step 
lower or higher pitch range (all other acoustic features held constant). Pitch was altered by the use of computer multimedia software, MAGIX Audio Studio 10 Deluxe. Chicks from both exposure conditions thus received a simultaneous choice test between the same two maternal calls, but one call had the familiarized pitch and the other call had a novel pitch. All other acoustic features of the call were unmodified and thus identical across the two variants of the call. The control group received the same test without having received any supplemental prenatal exposure.

Testing involved placing each chick in the arena midway between the two approach areas. All birds were given a 5-min simultaneous choice test between the two variants of the same bobwhite maternal call. Each call originated from one of the speakers located in each of the two approach areas. The sound intensity of each call was adjusted to approximately peak at $65 \mathrm{~dB}$, measured from the point where the chicks were placed in the arena. The locations of the two calls presented during testing were counterbalanced across trials to prevent possible side bias.

\section{Data Analysis}

The relevant dependent variables were the measures of preference for the auditory stimuli presented during the testing trials. The measures of preference used were 1) total number of entries into familiar versus modified call approach areas, 2) latency to approach familiar versus modified call approach areas, and 3) total duration of time spent within familiar versus modified call approach areas. Chicks failing to spend at least 30 seconds in an approach area were scored as non-responders. Chicks failing to spend at least twice as long in one approach area than the other were scored as having no preference. A chick was scored as showing a preference for a particular call if the chick 
spent at least 30 seconds in an approach area and at least twice as long in that approach area as the other.

Duration and latency scores within groups were evaluated using the Wilcoxon matched-pairs, signed-ranks test. Individual preferences were evaluated by the chi-square test. Significance levels of $\mathrm{p}<.05$ (two-tailed) were used to evaluate all results. Duration and latency scores for the two calls were converted into proportion of duration (PTD) and proportion of total latency (PTL) scores (duration/latency for the familiar divided by total duration/latency for both calls). A one-way analysis of variance was performed on the latency and duration measures to compare responses across groups.

\section{CHAPTER IV.}

\section{Experiment}

\section{Introduction}

To investigate the effects of sensory simulation on selective attention and prenatal perceptual learning, quail embryos were exposed prenatally to either unimodal or bimodal stimulation. In keeping with the predictions of the Intersensory Redundancy Hypothesis, I hypothesized that chicks receiving unimodal (auditory only) prenatal exposure to a maternal call would prefer that call over the same call with altered pitch. In contrast, I hypothesized that chicks receiving redundant bimodal (audio-visual) prenatal exposure to the maternal call would not prefer the familiar call over the same call with altered pitch. Naive chicks receiving no supplemental prenatal stimulation should also show no preference between the two variants of the call during postnatal testing. 


\section{Method}

One hundred and forty-one bobwhite quail embryos, divided into 2 experimental groups and 1 control group (unimodal $n=49$; bimodal $n=51$; control $n=41$ ), served as subjects. One group of embryos was exposed in groups of 10-12 to an individual maternal call altered to a one-step lower or higher pitch range (all other acoustic features held constant) for 10 min each hour for the $24 \mathrm{hr}$ period prior to hatch (Unimodal Group). The second group of embryos was exposed to the same maternal call altered to a one-step lower or higher pitch range (all other acoustic features held constant) synchronously paired with a pulsing light (Bimodal Group). The third group received no prenatal sensory stimulation (Naïve Control Group). All groups were tested at $24 \mathrm{hr}$ after hatching in a simultaneous choice test between the two individual variants of the maternal call (see General Methods for details).

\section{Results and Discussion}

Results are shown in Tables 1 and 2. These results indicate that when quail embryos are not exposed to any prenatal sensory stimulation (Naïve Control group) they do not prefer $\left(\chi^{2}=1.357, p=.507\right)$ either variant of the bobwhite maternal call during testing $24 \mathrm{hr}$ after hatching. This group also did not show significant differences in latency of approach or in the amount of time spent near either call. On the other hand, when quail embryos are prenatally exposed to auditory (unimodal) presentations of a maternal call they significantly prefer the familiarized call over a modified call with altered pitch at testing $24 \mathrm{hr}$ after hatching $\left(\chi^{2}=20.42, p<.000\right)$. Wilcoxon signed-ranks test revealed that the unimodal group showed significantly longer duration $(\mathrm{z}=-3.526$, $\mathrm{p}$ $=.000)$ and shorter latency $(\mathrm{z}=-2.789, \mathrm{p}=.005)$ scores for the familiarized call than the 
unfamiliarized call. Subjects that received prenatal audiovisual (bimodal) exposure to a maternal call failed to demonstrate a preference for either of the call variants at testing 24 hr after hatching $\left(\chi^{2}=0.326, p>0.85\right)$. The bimodal group also showed no significant differences in their duration to the two calls $(\mathrm{z}=-.700, \mathrm{p}=.484)$ or latency $(\mathrm{z}=-.543, \mathrm{p}=$ .587) scores. A one-way ANOVA demonstrated that the unimodal group had significantly larger PTD $(F(1,84)=12.148, p=.001)$ and lower latency (PTTA difference) scores $(\mathrm{F}(1,84)=4.310, \mathrm{p}=.041)$ to the familiar call than the bimodal group. The mean PTDT for both experimental conditions are shown in Figure 1.

Table 1: Preference Scores for Subjects Tested at 24 hr Following Hatching

\begin{tabular}{cccccc} 
Condition & n & $\begin{array}{c}\text { n- } \\
\text { responding }\end{array}$ & $\begin{array}{c}\text { Familiar } \\
\text { Call }\end{array}$ & $\begin{array}{c}\text { Unfamiliar } \\
\text { Call }\end{array}$ & $\begin{array}{c}\text { No } \\
\text { Preference }\end{array}$ \\
\hline $\begin{array}{c}\text { Naïve } \\
\text { Control }\end{array}$ & 41 & 28 & 7 & 12 & 9 \\
$\begin{array}{c}\text { Unimodal } \\
\text { (audio only) }\end{array}$ & 49 & 43 & $28^{*}$ & 5 & 10 \\
$\begin{array}{c}\text { Bimodal } \\
\text { (audiovisual) }\end{array}$ & 51 & 43 & 13 & 16 & 14 \\
\hline
\end{tabular}

${ }^{*} \mathrm{p}<.05$ (Chi-Square Test) 
Table 2: Duration Scores (in seconds) for Chicks in Simultaneous Choice Test

Condition Familiar Call Unfamiliar Call Difference

\begin{tabular}{cccc}
\hline Naive & 32.95 & 42.84 & Not significant \\
& $(56.19)$ & $(70.207)$ & \\
Unimodal & 64.72 & 17.55 & $<.05$ \\
(Audio only) & $(62.82)$ & $(47.59)$ & \\
& & & Not significant \\
Bimodal & 35.56 & 40.41 & \\
(Audio-visual) & $(58.03)$ & $(53.37)$ &
\end{tabular}

Median Scores are shown. Standard deviations are shown in parentheses.

Table 3: Latency Scores (in seconds) for Chicks in Simultaneous Choice Test

$\begin{array}{cccc}\text { Condition } & \text { Familiar Call } & \text { Unfamiliar Call } & \text { Difference } \\ & 50.92 & 38.84 & \text { Not significant } \\ \text { Naive } & (92.29) & (87.17) & \\ & & & \\ \text { Unimodal } & 49.66 & (111.58) & \\ \text { (Audio only) } & (52.28) & & \text { Not significant } \\ \text { Bimodal } & 32.31 & 42.41 & \\ \text { (Audio-visual) } & (73.55) & (80.17) & \end{array}$

Median Scores are shown. Standard deviations are shown in parentheses. 
Figure 1: Mean Proportion of Total Duration Time for the Familiar Call

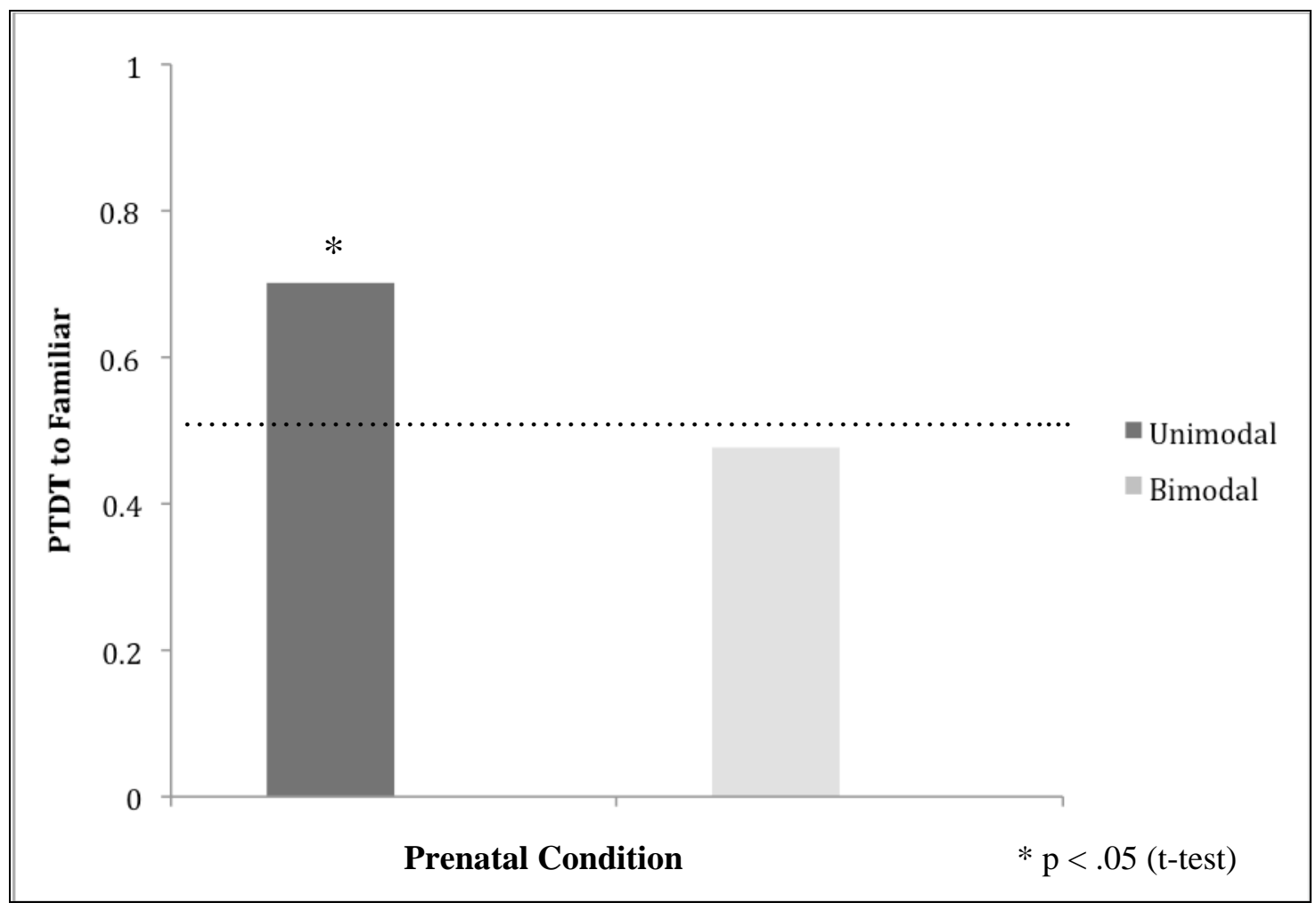

CHAPTER V.

General Discussion

The main goal of the present study was to explore the second prediction of the Intersensory Redundancy Hypothesis, which states that early in development information presented to a single sense modality selectively recruits attention to the modality-specific properties of stimulation and facilitates perceptual learning of those properties at the expense of amodal properties. According to the IRH, during unimodal stimulation there are no redundant amodal properties competing for attention, thus allowing the modalityspecific properties to be attended to and processed. The results of the current study support this prediction of the IRH, demonstrating that prenatal unimodal exposure 
facilitates learning of modality-specific properties of stimulation, whereas redundant bimodal stimulation interferes with attention to and learning of modality-specific properties of stimulation. Chicks significantly preferred a familiarized maternal call over the same call with altered pitch following prenatal unimodal auditory exposure, indicating detection of the pitch change. In contrast, following prenatal redundant audiovisual exposure, chicks failed to prefer the familiarized call over the modified call, indicating no detection of the pitch change. The chicks' preference for the familiar version of the maternal call suggests that they were attending to specific features of the call during prenatal stimulus presentations. The IRH model would suggest that the chicks' ability to perceive the pitch change is a result of their selective attention to the modality-specific feature of pitch during prenatal exposure.

A key question in the study of perceptual development is what causes certain properties of stimulation to be salient and attended to while other properties are disregarded. The IRH proposes that the saliency of certain properties over others selectively recruits attention to these properties during prenatal and postnatal development. Specifically, it is the redundancy of amodal information across sensory modalities that grabs the organism's attention. For instance, the sight and sound of a bouncing ball provide redundant information (e.g. tempo) that is common across both the visual and auditory modalities.

However, events also provide modality-specific information, and perception of these properties is undoubtedly crucial to typical perceptual development. In avian species, both songs and calls are important communicative tools, and thus, detection of modality-specific properties such as pitch is critical to perceptual and social development 
and learning. For example, in some species of birds such as the white-throated sparrow (Zonotrichia albicollis), certain note frequencies in the birds' vocalizations provide other members of its species clues to individual identity (Hurly, Ratcliffe, Weary, \& Weisman, 1992). Furthermore, male black-capped chickadees (Poecile atricapillus) have to produce a specific internote pitch alteration in order to attract and arouse females (Weisman \& Ratcliffe, 2004). In quail, which were used in the current study, calls help to maintain and regroup pairs and coveys, which is critical for defense against predators (Johnsgard, 1974). The detection of subtle changes such as pitch in calls and songs are, thus, critical skills for developing chicks and appear to emerge during prenatal development.

In human development, detection and processing of modality-specific properties of stimulation are also essential to perception and learning. Properties such as color and pattern allow an individual to differentiate between two different objects or individuals. Detection of pitch and timbre, both modality-specific properties, allow a person to distinguish between voices of different individuals. Effective social perception requires that persons learn to distinguish among numerous faces and relate those faces with specific voices. Hence, perceptual processing of amodal and modality-specific information is key to typical social development.

It is important to mention that quail embryos in the present study were exposed to stimuli not likely encountered in their natural environment. Although the patterned pulsing light allowed for a high level of experimental control, future studies should use stimuli considered to be more ecologically valid. For example, synchronized vestibular and auditory stimulation are likely more relevant in their natural setting since it resembles the vibratory sensation produced from the mother hen's vocalizations. Thus, using 
species-typical calls paired with vibration may be a more suitable method to further assess this topic. Nevertheless, the effect of unimodal facilitation was seen here despite having used unnatural stimuli, thereby demonstrating the generalizability of the developmental processes proposed by the IRH across different stimulus conditions.

The results of this study have important implications for the development of perception and cognition in young infants as well as across the lifespan. The IRH provides useful and testable predictions for understanding how perceptual learning is driven by selective attention. Studies such as the one presented here provide insight into how selective attention is recruited toward specific stimulus properties of events in early development. This study can also serve as a basis for future studies that test the developmental predictions of the IRH using the same modality-specific property, pitch. The third prediction suggests that as development proceeds, perceptual differentiation, processing efficiency, and flexibility of attention all increase allowing for detection of amodal and modality-specific properties in both redundant bimodal and nonredundant unimodal stimulation. Subsequently, the fourth prediction argues that across the lifespan, intersensory and unimodal facilitation occur in tasks in which the perceiver experiences high cognitive load and attentional resources are drained. In tasks considered difficult relative to the expertise of the perceiver, the degree of intersensory or unimodal facilitation is more pronounced. Thus, one practical approach for testing these predictions would involve providing similar unimodal and bimodal presentations of a maternal call to quail chicks postnatally, and subsequently testing them 24 hours later in a simultaneous choice test between the familiarized call and the same call with altered pitch (using the same pitch alterations utilized in this study). A second experiment would involve 
narrowing the pitch change between the two variants of the call, thereby increasing the difficulty of the task. According to the predictions of the IRH, older quail chicks should be able to detect the pitch change used in the present study under conditions of both unimodal and bimodal stimulation. However, when the task is perceived to be more difficult (narrower pitch change), the same aged chicks should demonstrate unimodal facilitation. Such studies would provide clues as to how detection of stimulus properties change as an individual develops. Furthermore, how perceptual learning develops with experience is fundamental to comprehending processes of typical and atypical cognitive and social development. 


\section{REFERENCES}

Bahrick, L.E. (1983). Infants' perception of substance and temporal synchrony in multimodal events. Infant Behavior \& Development, 6, 429-451.

Bahrick, L.E. (1987). Infants' intermodal perception of two levels of temporal structure in natural events. Infant Behavior and Development, 10, 387-416.

Bahrick, L.E. (1988). Intermodal learning in infancy: Learning on the basis of two kinds of invariant relations in audible and visible events. Child Development, 59, 197209.

Bahrick, L.E. (1992). Infants' perceptual differentiation of amodal and modality-specific audiovisual relations. Journal of Experimental Child Psychology, 53, 180-199.

Bahrick, L.E. (1994). The development of infants' sensitivity to arbitrary intermodal relations. Ecological Psychology, 6, 111-123.

Bahrick, L.E. (2001). Increasing specificity in perceptual development: Infants’ detection of nested levels of multimodal stimulation. Journal of Experimental Child Psychology, 79, 253-270.

Bahrick, L.E., Flom, R., \& Lickliter, R. (2002). Intersensory redundancy facilitates discrimination of tempo in 3-month-old infants. Developmental Psychobiology, 41, 352-363.

Bahrick, L.E., \& Lickliter, R. (2000). Intersensory redundancy guides attentional selectivity and perceptual learning in infancy. Developmental Psychology, 36, 190-201.

Bahrick, L.E., \& Lickliter, R. (2002). Intersensory redundancy guides early cognitive and perceptual development. In R. Kail \& H. Reese (Eds.), Advances in child development and behavior (Vol. 30, pp. 153-187). New York: Academic Press.

Bahrick, L.E., \& Lickliter, R. (in press). The role of intersensory redundancy in early perceptual, cognitive, and social development. In: A. Bremner, D. J. Lewkowicz, \& C. Spence (Eds.), Multisensory development. Oxford University Press.

Bahrick, L.E., Lickliter, R., \& Flom, R. (2006). Up versus down: The role of intersensory redundancy in the development of infants' sensitivity to the orientation of moving objects. Infancy, 9, 73-96.

Bahrick, L.E., Lickliter, R., Vaillant, M., Shuman, M., \& Castellanos, I. (2004, May). 
Infant discrimination of faces in the context of dynamic multimodal events: Predictions from the intersensory redundancy hypothesis. Poster session presented at the International Conference on Infant Studies, Chicago, IL.

Bahrick, L.E., Netto, D., \& Hernandez-Reif, M. (1998). Intermodal perception of adult and child faces and voices by infants. Child Development, 69, 1263-1275.

Bahrick, L.E., \& Pickens, J. (1994). Amodal relations: The basis for intermodal perception and learning in infancy. In D. J. Lewkowicz \& R. Lickliter (Eds.), The development of intersensory perception: Comparative perspectives (pp. 205-233). Hillsdale, NJ: Erlbaum.

Flom, R., \& Bahrick, L.E. (2007). The development of infant discrimination of affect in multimodal and unimodal stimulation: The role of intersensory redundancy. Developmental Psychology, 43, 238-252.

Gibson, E. (1969). Principles of perceptual learning. New York: Appleton-CenturyCrofts.

Gibson, E.J. \& Pick, A.D. (2000). An ecological approach to perceptual learning and development. Oxford University Press.

Gottlieb, G. (1971). Ontogenesis of sensory function in birds and mammals. In E. Tobach, L. R. Aronson, \& E. Shaw (Eds.), The biopsychology of development (pp. 67-128). New York: Academic Press.

Gottlieb, G. (1993). Social induction of malleability in ducklings: Sensory basis and psychological mechanism. Animal Behaviour, 45, 707-719.

Honeycutt, H., \& Lickliter, R. (2002). Prenatal experience and postnatal perceptual preferences: Evidence for attentional-bias in bobwhite quail embryos (Colinus virginianus). Journal of Comparative Psychology, 116, 270-276.

Hurly, T.A., Ratcliffe, L., Weary, D.M., \& Weisman, R. (1992). White-throated sparrows (Zonotrichia albicollis) can perceive pitch change in conspecific song by using the frequency ration independent of the frequency difference. Journal of Comparative Psychology, 106(4), 388-391.

Johnsgard, P. A. (1974). Quail music: The complex calls of a bird contain clues to its evolution. Natural History, 83(3), 34-40.

Lewkowicz, D.J. (1988a). Sensory dominance in infants: 1. Six-month-old infants' response to auditory-visual compounds. Developmental Psychology, 24, 155171. 
Lewkowicz, D.J. (1988b). Sensory dominance in infants: 2. Ten-month-old infants' response to auditory-visual compounds. Developmental Psychology, 24, 172182.

Lewkowicz, D.J. (1992). Responsiveness to auditory and visual components of a sounding/moving compound stimulus in human infants. Perception and Psychophysics, 52, 519-528.

Lewkowicz, D.J. (1994). Limitations on infants' response to rate-based auditory-visual relations. Developmental Psychology, 30, 880-892.

Lewkowicz, D.J. (1996). Infants' response to the audible and visible properties of the human face: Role of lexical syntactic content, temporal synchrony, gender, and manner of speech. Developmental Psychology, 32, 347-366.

Lewkowicz, D.J. (2000). The development of intersensory temporal perception: An epigenetic systems/limitations view. Psychological Bulletin, 126, 281-308.

Lewkowicz, D.J., \& Lickliter, R. (1994a). The development of intersensory perception: Comparative perspectives. Hillsdale, NJ: Erlbaum.

Lewkowicz, D.J., \& Lickliter, R. (1994b). Insights into mechanisms of intersensory development: The value of a comparative, convergent-operations approach. In D. J. Lewkowicz \& R. Lickliter (Eds.), The development of intersensory perception: Comparative perspectives (pp. 403-413). Hillsdale, NJ: Erlbaum.

Lickliter, R. (2005). Prenatal sensory ecology and experience: implications for perceptual and behavioral development in precocial birds. Advances in the Study of Behavior, 35, 235-274.

Lickliter, R. (2000). The role of sensory stimulation in perinatal development: Insights from comparative research for care of the high-risk infant. Journal of Developmental \& Behavioral Pediatrics, 21, 437-447.

Lickliter, R., \& Bahrick, L.E. (2000). The development of infant intersensory perception: Advantages of a comparative, convergent-operations approach. Psychological Bulletin, 126, 260-280.

Lickliter, R., Bahrick, L.E., \& Honeycutt, H. (2002). Intersensory redundancy facilitates perceptual learning in bobwhite quail embryos. Developmental Psychology, 38, $15-23$.

Lickliter, R., Bahrick, L.E., \& Honeycutt, H. (2004). Intersensory redundancy enhances memory in bobwhite quail embryos. Infancy, 5, 253-269. 
Lickliter, R., \& Lewkowicz, D. J. (1995). Intersensory experience and early perceptual development: Attenuated prenatal sensory stimulation affects postnatal auditory and visual responsiveness in bobwhite quail chicks (Colinus virginianus). Developmental Psychology, 31, 609-618.

Lickliter, R., \& Stoumbos, J. (1991). Enhanced prenatal auditory experience facilitates species-specific visual responsiveness in bobwhite quail chicks (Colinus virginianus). Journal of Comparative Psychology, 105, 89-94.

Morrongiello, B.A. (1994). Effects of co-location on auditory-visual interactions and cross-modal perception in infants. In D. J. Lewkowicz \& R. Lickliter (Eds.), The development of intersensory perception: Comparative perspectives (pp. 235-263). Hillsdale, NJ: Erlbaum.

Rose, S.A., \& Ruff, H.A. (1987). Cross-modal abilities in human infants. In J. Osofsky (Ed.), Handbook of infant development (pp. 318-362). New York: Wiley.

Spelke, E. (1979). Perceiving bimodally specified events in infancy. Developmental Psychology, 15, 626-636.

Vaillant-Molina, M., Newell, L., Castellanos, I., Bahrick, L.E., \& Lickliter, R. (2006, June). Intersensory redundancy impairs face perception in early development. Presented at the International Conference for Infant Studies, Kyoto, Japan.

Walker, A.S. (1982). Intermodal perception of expressive behaviors by human infants. Journal of Experimental Child Psychology, 33, 514-535.

Walker-Andrews, A.S., Bahrick, L.E., Raglioni, S.S. \& Diaz, I. (1991). Infants' bimodal perception of gender. Ecological Psychology, 3, 55-75.

Weisman, R., Ratcliffe, L. (2004). Relative pitch and the song of black-capped chickadees. American Scientist, 92, 532-539. 